\title{
Non-Gaussian states produced by close-to-threshold optical parametric oscillators: Role of classical and quantum fluctuations
}

\author{
V. D’Auria, ${ }^{1, *}$ C. de Lisio, ${ }^{2,3}$ A. Porzio,,${ }^{2,4, \dagger}$ S. Solimeno,,${ }^{3,4}$ Javaid Anwar, ${ }^{5}$ and M. G. A. Paris ${ }^{6,7, \ddagger}$ \\ ${ }^{1}$ Laboratoire Kastler Brossel, Université Pierre et Marie Curie, Ecole Normale Supérieure, Centre National \\ de la Recherche Scientifique, 4 place Jussieu, F-75252 Paris, France \\ ${ }^{2}$ CNR-SPIN, Monte Sant'Angelo, via Cintia, I-80126 Naples, Italy \\ ${ }^{3}$ Dipartimento di Scienze Fisiche, Università “Federico II," Monte Sant'Angelo, via Cintia, I-80126 Naples, Italy \\ ${ }^{4}$ Consorzio Nazionale Interuniversitario per le Scienze Fisiche della Materia UdR Napoli Università, \\ Monte Sant'Angelo, via Cintia, I-80126 Naples, Italy \\ ${ }^{5}$ Department of Physics, COMSATS Institute of Information Technology, Islamabad, Pakistan \\ ${ }^{6}$ Dipartimento di Fisica, Università degli studi di Milano, I-10133 Milan, Italy \\ ${ }^{7}$ Consorzio Nazionale Interuniversitario per le Scienze Fisiche della Materia UdR Milano Università, I-20133 Milan, Italy
}

(Received 22 July 2009; published 29 March 2010)

\begin{abstract}
Quantum states with non-Gaussian statistics generated by optical parametric oscillators (OPO) with fluctuating parameters are studied by means of the kurtosis excess of the external field quadratures. The field generated is viewed as the response of a nonlinear device to the fluctuations of pump laser amplitude and frequency, crystal temperature, and cavity detuning, in addition to quantum noise sources. The kurtosis excess has been evaluated perturbatively up to the third order in the strength of the crystal nonlinear coupling factor and the second order in the classical fluctuating parameters. Applied to the device described in Opt. Expr. 13, 948 (2005), the model has given values of the kurtosis excess in good agreement with the measured ones.
\end{abstract}

DOI: 10.1103/PhysRevA.81.033846

PACS number(s): 42.65.Yj, 03.67.Bg, 42.50.Dv, 42.50.Lc

\section{INTRODUCTION}

Non-Gaussian (NG) resources (i.e., NG states and/or operations) [1-6] are required for realizing relevant quantum information protocols as, for example, entanglement distillation and swapping [7-10]. It has been demonstrated that they may improve the fidelity of teleportation [11-13] and cloning [14], and NG states are more effective in revealing nonlocality [15-18]. Thus the reliable generation of NG singleand two-mode states assumes a relevant role. Recently, a few schemes to generate NG states based on conditional de-Gaussification protocols have been proposed [11-13] and realized $[19,20]$. Moreover, it has been proven that phase diffusing a squeezed vacuum state makes it a NG state [4-6].

Departures from Gaussian statistics have been observed (see Ref. [21]) in the outcomes for the field quadrature $X_{\theta}=$ $\left(e^{-i \theta} a+e^{i \theta} a^{\dagger}\right) / 2$, outing a degenerate optical parametric oscillator (OPO). These deviations have been quantified by measuring for different operating conditions the departure of the fourth moment $\left\langle X_{\theta}^{4}\right\rangle$ from its Gaussian value. In particular, a dependence on the quadrature phase $\theta$ has been observed, with the maximum departure always appearing in correspondence with the antisqueezed amplitude quadrature $(\theta=0)$.

No doubt the observed deviations are because of a nonlinear response of the device to Gaussian quantum and classical fluctuating parameters such as pump laser amplitude and phase, cavity length, and OPO crystal temperature. While in the linear analysis, only the quantum input noise contributes to

\footnotetext{
*virginia.dauria@spectro.jussieu.fr

†alberto.porzio@na.infn.it

${ }^{\ddagger}$ matteo.paris@fisica.unimi.it
}

the OPO output signal, by expanding the equation of motion to higher orders, classical fluctuations contribute to the output as well. As a result of the multiplicative mixing of several noise sources, the signal loses its Gaussian character. The weight of these sources in determining the deviation from the Gaussian statistics was not clear at the time of publication of Ref. [21]. This article is meant to present and discuss a consistent theoretical framework to explain and interpret the observed experimental evidences.

OPOs rely on parametric down-conversion: A strong pump beam at frequency $\omega_{p}$ interacts in a nonlinear crystal with the vacuum fields, thus generating two beams, signal and idler, at frequencies $\omega_{s}$ and $\omega_{i}$, respectively [22-24]. This mechanism is represented in the Hamiltonian by the product of three field operators, $a_{p}$ (pump), $a_{s}$ (signal), and $a_{i}$ (idler), and is described dynamically by Langevin equations. The model herein presented starts by including in the Graham and Haken Langevin equations (GHLE) different classical noise sources. Then, expanding $a_{s, i}$ as a power series in the strengths of the quantum and classical fluctuations, a hierarchy of Langevin equations is obtained in which the field at a given order acts as a source for the next one. This has been done up to the third order, thus culminating with Fig. 5 showing an adequate agreement of the computed difference (kurtosis excess) $K_{\theta}=\left(\left\langle X_{\theta}^{4}\right\rangle-\right.$ $\left.3\left\langle X_{\theta}^{2}\right\rangle^{2}\right) /\left\langle X_{\theta}^{2}\right\rangle^{2}$ with the measured values $K_{\theta}$ of Ref. [21].

A perturbative analysis of an ideal OPO was already developed in Ref. [25]: According to this article, nonlinear contributions become comparable to the linear output only at a relative distance from the threshold of about $\sim 10^{-6}$, while the data of Ref. [21] were measured at a distance of $\sim 5 \times 10^{-2}$, signaling the occurrence of more complex mechanisms. Hence it has been essential to account for contributions from different fluctuating parameters, each represented as a Gaussian process weighted by its standard deviations $g_{i}$. For the sake of generality, the GHLE [26] model has been developed for a 
nondegenerate OPO, whereas the field moments have been calculated for a degenerate OPO to allow a direct comparison with Ref. [21].

Different-order fields have been represented as the convolution of the previous-order field with a $2 \times 2$ matrix $\mathbf{G}$ which becomes singular on approaching the threshold. This singularity is at the origin of the enhancement of higher-order effects in the critical region.

The article is organized as follows. In Sec. II, the extended GHLE model for an OPO with fluctuating parameters is introduced and discussed. The squeezing of the intracavity field is discussed in Sec. III. Section IV deals with the statistics of the quadratures outside the cavity as measured by a finite bandwidth detector. The deviation from the Gaussian statistics in terms of $K_{\theta}$ is analyzed by dwelling on the agreement of the results provided by the model with the experimental findings. Plots of $K_{\theta}$ versus $\theta$ and $K_{\theta}$ for $\theta=0$ versus $E^{2}$ are reported. Section $\mathrm{V}$ closes the article with concluding remarks. Details about the linearization, together with a few analytical derivations, are reported in the appendixes.

\section{LANGEVIN EQUATIONS}

Consider the set of the three OPO cavity modes $a_{k}\left(a_{p}=\right.$ $e^{-i \omega_{p} t-i \phi_{p}} a_{0}$ pump mode at frequency $\omega_{p}$ and phase $\phi_{p}$, with $a_{s}=e^{-i \omega_{s} t-i \frac{1}{2} \phi_{p}} a_{1}$ and $a_{i}=e^{-i \omega_{i} t-i \frac{1}{2} \phi_{p}} a_{2}$ being, respectively, signal and idler modes with $\omega_{p}=\omega_{s}+\omega_{i}$ ) whose mutual interaction under the action of a driving field $e^{-i \omega_{p} t} \mathcal{E}$ is described by the Hamiltonian

$$
H_{\text {int }}=i \hbar \frac{\chi}{2}\left(a_{1} a_{2} a_{0}^{\dagger}-a_{0} a_{1}^{\dagger} a_{2}^{\dagger}\right)+i \hbar\left(\mathcal{E}^{*} a_{0}-\mathcal{E} a_{0}^{\dagger}\right),
$$

where $\chi$ is the coupling parameter proportional to the crystal second-order susceptibility $\chi^{(2)}$ since real lasers are characterized by a field $\mathcal{E}=\epsilon\left(1+g_{\mu_{p}} \hat{\mu}_{p}\right) e^{-i \phi_{p}}$ of constant amplitude $\epsilon$ modulated by a fluctuating factor $1+g_{\mu_{p}} \mu_{p}(t)$ $\left(\left\langle\mu_{p}\right\rangle=0\right)$ times a phase factor $e^{-i \phi_{p}}$, where $\phi_{p}(t)$ is a slowly diffusing phase, that is, $\left\langle\left[\phi_{p}(t)-\phi_{p}\left(t^{\prime}\right)\right]\right\rangle^{2}=\Delta_{\ell}\left|t-t^{\prime}\right|$, with $\Delta_{\ell}$ being the laser linewidth [24].

The cavity modes are characterized by damping factors $\gamma_{k, M}, \gamma_{k, x}\left(\gamma_{k}=\gamma_{k, M}+\gamma_{k, x}\right)$ because of the output mirror $(M)$ and the other loss mechanisms ( $x$, crystal absorption and scattering, absorption of the two mirrors, etc.), respectively. The evolution of the cavity-mode operators can be described by the GHLE [26]:

$$
\begin{aligned}
& \dot{a}_{j}=-\left(\gamma_{j}-i v_{j}-i \frac{1}{2} \dot{\phi}_{p}\right) a_{j}+\frac{\chi}{2} a_{0} a_{j^{\prime}}^{\dagger}+e^{-i g_{\varpi_{p}} \phi_{p} / 2} R_{j}, \\
& j=1,2 \text { and } j \neq j^{\prime} \\
& \dot{a}_{0}=-\left(\gamma_{0}-i \nu_{0}-i \dot{\phi}_{p}\right) a_{0}-\frac{\chi^{*}}{2} a_{1} a_{2}+\epsilon\left(1+g_{\mu_{p}} \mu_{p}\right) \\
&+e^{i g_{\sigma_{p}} \phi_{p}} R_{0},
\end{aligned}
$$

where $R_{k}(t)=\sqrt{2 \gamma_{k, M}} b_{k, M}+\sqrt{2 \gamma_{k, x}} b_{k, x}$ takes into account the $\delta$-correlated vacuum fluctuations, $\left\langle b_{k, M, x}(t) b_{k, M, x}^{\dagger}\left(t^{\prime}\right)\right\rangle=$ $\delta\left(t-t^{\prime}\right)$, entering the OPO cavity. Modes are assumed to be slightly detuned by $v_{k}=\pi c / L_{k}\left[L_{k}\left(\omega_{k} / \pi c\right)\right]-\omega_{k}$, with $L_{k}$ being the OPO optical length at frequency $\omega_{k}$ and $[x]$ being the closest integer to $x$.

In the following, we will indicate by $\kappa_{k}=\gamma_{k}-i \nu_{k}=$ $\left|\kappa_{k}\right| e^{-i \psi_{k}}\left(\right.$ with $\psi_{1}=\psi_{2}$ ) the complex damping coefficients, by $\kappa=\left|\kappa_{1}+\kappa_{2}\right| / 2$ the mean decay rate, and by $\tau=\kappa t$ the time normalized to the cavity lifetime $\kappa^{-1}$. A caret will mark quantities normalized to $\kappa$ (e.g., $\hat{\epsilon}=\epsilon / \kappa)$ and a tilde those such that the integral of their correlation function [e.g., $\left.\left\langle\hat{\mu}_{p}(\tau) \mu_{p}\left(\tau^{\prime}\right)\right\rangle=C_{\mu_{p}}\left(\tau-\tau^{\prime}\right)\right]$ is equal to 1 [e.g., $\left.\int_{-\infty}^{\infty} C_{\mu_{p}}(\tau) d \tau=1\right]$. In particular, the Gaussian $\delta$-correlated process $d \phi_{p} / d t$ will be replaced by $d \phi_{p} / d \tau=g_{\varpi_{p}} \varpi_{p}$ with $\left\langle\varpi_{p}(\tau) \varpi_{p}\left(\tau^{\prime}\right)\right\rangle=\delta\left(\tau-\tau^{\prime}\right)$ and $g_{\varpi_{P}}=\sqrt[4]{\left\langle\hat{\Delta}_{\ell}^{2}\right\rangle}$.

In real devices, beside the fluctuations related to classical noise of the laser beam, the parameters $v_{k}$ and $\chi$ of Eq. (2) experience also the effects of mechanical vibrations. Residual fluctuations of the cavity optical length $\delta L_{k}$, at frequency $\omega_{k}$, induce deviations $\delta v_{k}=-\left(\delta L_{k} / L_{k}\right) \omega_{k}$ of the mode detunings from their average values $\left\langle v_{k}\right\rangle$. Usually, an active control guarantees that the standard deviation of $\delta v_{0}$ is a small fraction of $\gamma_{0}$.

The parameter $\chi$ is proportional to the crystal susceptibility $\chi^{(2)}$ through the Boyd-Kleinman function $H_{\mathrm{BK}}(\sigma, \varkappa, \xi)$ [27] of the phase-matching factor $\sigma$, the focusing parameter $\xi$, and the crystal absorption $\varkappa$. Variable $\sigma(T)$ depends on the crystal temperature $T$ through the refractive indices at the interaction wavelengths. If the cavity configuration is far from the concentric one, the dependence of $\xi$ on the cavity geometry fluctuations can be neglected. Under this assumption, $\chi$ will be replaced in the system (2) by $\bar{\chi} e^{-i \phi_{\chi}}\left(1+g_{T} \delta \hat{T}\right)$ with $\bar{\chi}$ depending on the slow variations of $T$ while $\phi_{\chi}$ is a phase depending on the position of the beam waist with respect to the crystal center. With an accurate alignment, $\phi_{\chi}$ can be set equal to 0 . Variable $g_{T}$ is defined by

$$
g_{T}=\sqrt{\left\langle\delta T^{2}\right\rangle} \frac{d \log H_{\mathrm{BK}}}{d T} .
$$

In conclusion, the OPO analyzed in the following is characterized by four classical fluctuating parameters $g_{\mu_{p}} \hat{\mu}_{p}$, $g_{\varpi_{p}} \varpi_{p}, g_{\nu_{k}} \delta \hat{v}$, and $g_{T} \delta \hat{T}$, where $\hat{\mu}_{p}, \varpi_{p}, \delta \hat{v}$, and $\delta \hat{T}$ are Gaussian processes with unit standard deviations and $g_{\mu_{p}}, g_{\varpi_{p}}, g_{v_{k}}\left\{=\left[\left(\delta L_{k} / L_{k}\right) /\left(\delta L_{0} / L_{0}\right)\right]\left(\omega_{k} / \omega_{0}\right) g_{\nu_{0}}\right\}$, and $g_{T}$ are the corresponding weights. These four terms, together with $g_{\chi}=|\bar{\chi}| /\left(2\left|\kappa_{0} \kappa\right|\right)^{1 / 2}$, describing the nonlinear interaction of strength $\bar{\chi}$ [25], determine the OPO dynamics. For typical operating conditions $\left(g_{\chi} \simeq 10^{-6}, \kappa \simeq 10-20 \mathrm{MHz}, \sqrt{\left\langle\Delta_{\ell}^{2}\right\rangle} \simeq\right.$ $1-1000 \mathrm{~Hz}, \sqrt{\left\langle\delta T^{2}\right\rangle} \simeq 1-10 \mathrm{mK}$, and $\left.\partial n / \partial T \approx 10^{-6}-10^{-4}\right)$, $g_{\mu_{p}}, g_{\varpi_{p}}, g_{v_{k}}$, and $g_{T}$ range in the intervals $10^{-2}-10^{-1}$, $10^{-4}-10^{-2}, 10^{-5}-10^{-1}$, and $10^{-5}-10^{-4}$, respectively.

The extended GHLE system (2) may now be written as

$$
\begin{aligned}
\dot{a}_{j}= & -\left(\hat{\kappa}_{j}-i \frac{g_{\varpi_{p}} \varpi_{p}}{2}+i g_{v_{j}} \delta \hat{v}\right) a_{j} \\
& +\sqrt{\frac{\left|\hat{\kappa}_{0}\right|}{2}}\left(1+g_{T} \delta \hat{T}\right) g_{\chi} a_{0} a_{j^{\prime}}^{\dagger}+e^{-i g_{\varpi_{p}} \phi_{p} / 2} \hat{R}_{j} \\
\dot{a}_{0}= & -\left(\hat{\kappa}_{0}-i g_{\varpi_{p}} \varpi_{p}+i g_{v_{0}} \delta \hat{v}\right) a_{0}-\sqrt{\frac{\left|\hat{\kappa}_{0}\right|}{2}}\left(1+g_{T} \delta \hat{T}\right) \\
& \times g_{\chi} a_{1} a_{2}+\hat{\epsilon}\left(1+g_{\mu_{p}} \hat{\mu}_{p}\right)+e^{i g_{\varpi_{p}} \phi_{p}} \hat{R}_{0} .
\end{aligned}
$$

A dot indicates derivatives with respect to $\tau$.

The OPO admits a threshold value for the amplitude $\epsilon=$ $\epsilon^{t h}=\left|\kappa_{0}\right| \sqrt{\left|\kappa_{1} \kappa_{2}\right|} /(2|\bar{\chi}|)$. Below threshold, the mode $a_{0}$ has a nonzero mean value $r_{0}$, which is related to the driving field amplitude $\hat{\epsilon}\left(\hat{\epsilon}=\hat{\kappa}_{0} r_{0}\right)$. Therefore, separating the average part 
$r_{0}$ from the fluctuating one $\delta a_{0}=r_{0} \alpha_{0}$, we put $a_{0}=r_{0}(1+$ $\alpha_{0}$ ), where $\alpha_{0}=\rho_{0}-i \varphi_{0}$. Conversely the modes $a_{j}$ have zero mean value and will be expressed in terms of rescaled operators $a_{j}=r_{j} \alpha_{j}$, with the $r_{j}$ defined in terms of $r_{0}$ :

$$
\left|\hat{\kappa}_{j}\right| r_{j}^{2}=\left|\hat{\kappa}_{0}\right| r_{0}^{2} .
$$

Passing now from the amplitudes $a_{k}$ to the scaled quantities $\alpha_{k}$, the extended GHLE [Eq. (3)] is rewritten as

$$
\begin{gathered}
\dot{\alpha}_{j}+\hat{\kappa}_{j} \alpha_{j}=E\left|\hat{\kappa}_{j}\right| \alpha_{j^{\prime}}^{\dagger}+g_{\chi} \hat{N}_{\chi_{j}}+\left|\hat{\kappa}_{j}\right| E\left(g_{\hat{T}} \delta \hat{T}+\alpha_{0}\right) \alpha_{j^{\prime}}^{\dagger} \\
+i\left(g_{\varpi_{p}} \varpi_{p} / 2-g_{v_{j}} \delta \hat{v}\right) \alpha_{j}+\left|\hat{\kappa}_{j}\right| E g_{\hat{T}} \delta \hat{T} \alpha_{0} \alpha_{j^{\prime}}^{\dagger} \\
\dot{\alpha}_{0}+\hat{\kappa}_{0} \alpha_{0}=-E\left|\hat{\kappa}_{0}\right| \alpha_{1} \alpha_{2}+g_{\chi} \hat{N}_{\chi_{0}}+\hat{\kappa}_{0} g_{\mu_{p}} \hat{\mu} p \\
+i g_{\varpi_{p}} \varpi_{p}-i g_{\nu_{0}} \delta \hat{v}+i g_{\varpi_{p}} \varpi_{p} \alpha_{0} \\
-i g_{v_{0}} \delta \hat{v} \alpha_{0}-E\left|\hat{\kappa}_{0}\right| g_{T} \delta \hat{T} \alpha_{1} \alpha_{2}
\end{gathered}
$$

where

$$
E=\frac{g_{\chi}}{\sqrt{2 \hat{\kappa}_{0} \hat{\kappa}_{1} \hat{\kappa}_{2}}} \hat{\epsilon} e^{i \psi_{0}}
$$

The preceding equations describe the dynamics of the fluctuating fields $\alpha_{k}$ as responses to the classical noise sources $\hat{\mu}_{p}$, $\varpi_{p}, \delta \hat{v}$, and $\delta \hat{T}$ and quantum terms

$$
\begin{gathered}
\hat{N}_{\chi_{j}}=\hat{R}_{j} e^{-i g_{\varpi_{p}} \phi_{p} / 2} /\left(g_{\chi} r_{j}\right), \\
\hat{N}_{\chi_{0}}=\hat{R}_{0} e^{i g_{\sigma_{p}} \phi_{p}} /\left(g_{\chi} r_{0}\right) .
\end{gathered}
$$

The system (5) may be solved perturbatively on expanding the field amplitudes as

$$
\alpha_{k}=\sum_{m=1}^{3} \alpha_{k}^{(m)}
$$

The first-order terms $(m=1)$ correspond to the linearized system; $\alpha_{k}^{(m \geqslant 2)}$ are generated by nonlinear sources $s_{k}^{(m)}$ made of a quantum contribution, proportional to $g_{\chi}^{m}$, and of mixed terms involving products of quantum and classical fluctuations of the type $g_{\chi}^{m-1} g_{i}$ and $g_{\chi}^{m-2} g_{i} g_{j}$. Any $s_{k}^{(m)}$ involves fields calculated up to the $(m-1)$ th order. By substituting the $\alpha_{k}$ expansion into Eq. (5) and grouping the terms corresponding to the same perturbative order, each $\alpha_{k}^{(m)}$ can be calculated as the convolution of the components $G_{k k^{\prime}}$ of the Green's matrix [see Eq. (A1)] relative to the linearized system, with $s_{k}^{(m)}$ and $s_{k^{\prime}}^{(m) \dagger}$.

For a degenerate (the signal and idler fields collapse into a single field in this case) and tuned $\left(\bar{v}_{k}=0\right)$ OPO, the extended GHLE [Eq. (5)] reduces to

$$
\begin{aligned}
\dot{\alpha}+\hat{\kappa} \alpha= & E \alpha^{\dagger}+g_{\chi} \hat{N}_{\chi}+E\left(g_{\tilde{T}} \delta \tilde{T}+\alpha_{0}\right) \alpha^{\dagger} \\
+ & i\left(\frac{1}{2} g_{\varpi_{p}} \varpi_{p}-g_{v_{j}} \delta \hat{v}\right) \alpha+E g_{\tilde{T}} \delta \hat{T} \alpha_{0} \alpha^{\dagger}, \\
\dot{\alpha}_{0}+\hat{\kappa}_{0} \alpha_{0}= & g_{\chi} \hat{N}_{\chi_{0}}+\hat{\kappa}_{0} g_{\mu_{p}} \hat{\mu}_{p}+i g_{\varpi_{p}} \varpi_{p}-i g_{v_{0}} \delta \hat{v} \\
& -\frac{E}{2}\left|\hat{\kappa}_{0}\right| \alpha^{2}+i g_{\varpi_{p}} \varpi_{p} \alpha_{0}-i g_{\nu_{0}} \delta \hat{v} \alpha_{0} \\
& -E\left|\hat{\kappa}_{0}\right| g_{T} \delta \hat{T} \alpha^{2}
\end{aligned}
$$

where the parameter

$$
E=\frac{2 g_{\chi}}{\sqrt{2 \hat{\kappa}_{0}}} \hat{\epsilon}=e^{i \psi_{0} / 2} \frac{\hat{\epsilon}}{\hat{\epsilon}^{t h}}=e^{i \psi_{0} / 2}|E|
$$

now represents the excitation $\hat{\epsilon}$ normalized to the threshold $\hat{\epsilon}^{t h}=\sqrt{\left|\hat{\kappa}_{0}\right| / 2} / g_{\chi}$ while $\hat{N}_{\chi}(\tau), \hat{N}_{\chi_{0}}(\tau)$, and $\varpi_{p}(\tau)$ are $\delta$-correlated processes:

$$
\begin{aligned}
\left\langle\hat{N}_{\chi}(\tau) \hat{N}_{\chi}^{\dagger}\left(\tau^{\prime}\right)\right\rangle & =\frac{4}{|E|^{2}} \delta\left(\tau-\tau^{\prime}\right), \\
\left\langle\hat{N}_{\chi_{0}}(\tau) \hat{N}_{\chi_{0}}^{\dagger}\left(\tau^{\prime}\right)\right\rangle & =\frac{4\left|\hat{\kappa}_{0}\right|^{2}}{|E|^{2}} \delta\left(\tau-\tau^{\prime}\right) .
\end{aligned}
$$

The correlation times for $\hat{\mu}_{p}$ are typically of the order of $0.2-1 \mu \mathrm{s}$, while those for $\delta \hat{v}$ and $\delta \hat{T}$ are of the order of milliseconds and seconds, respectively. Although $\delta \hat{v}$ and $\delta \hat{T}$ can be treated adiabatically, we have preferred to treat the noise sources in a unified fashion.

\section{A. Nonlinear terms for a degenerate OPO}

Expanding Eq. (5) up to the third order, the fields $\alpha^{(m)}$ (signal idler) and $\alpha_{0}^{(m)}$ (pump) of Eq. (7), represented in the vector form $\alpha^{(m)}=\left(\alpha^{(m)}, \alpha^{(m) \dagger}\right)^{T}, \alpha_{0}^{(m)}=\left(\alpha_{0}^{(m)}, \alpha_{0}^{(m) \dagger}\right)^{T}$, are given by

$$
\begin{gathered}
\alpha^{(m)}(t)=\int^{t} \mathbf{G}(t-\tau) \cdot \mathbf{s}^{(m)}(\tau) d \tau, \\
\alpha_{0}^{(m)}(t)=\int^{t} \mathbf{G}_{0}(t-\tau) \cdot \mathbf{s}_{0}^{(m)}(\tau) d \tau,
\end{gathered}
$$

with $\mathbf{G}$ and $\mathbf{G}_{0}$ defined by Eq. (A2), while the signal $\mathbf{s}^{(m)}$ and pump $\mathbf{s}_{0}^{(m)}$ sources read, respectively,

$$
\begin{gathered}
\mathbf{s}^{(1)}(\tau)=g_{\chi} \mathbf{N}_{\chi}(\tau), \\
\mathbf{s}^{(2)}(\tau)=\mathbf{B}^{(1)}(\tau) \cdot \alpha^{(1)}(\tau), \\
\mathbf{s}^{(3)}(\tau)=B^{(2)} \alpha^{(1) \dagger}(\tau), \\
\int^{\tau}\left(g_{\chi}^{2} \delta B^{(2)}\left(\tau-\tau^{\prime}\right) \mathbf{1}+\mathbf{B}^{(1,1)}\left(\tau-\tau^{\prime}\right)\right) \cdot \alpha^{(1)}\left(\tau^{\prime}\right) d \tau^{\prime}, \\
\mathbf{s}_{0}^{(1)}(\tau)=g_{\chi} \hat{\mathbf{N}}_{\chi_{0}}(\tau)+i\left[g_{\varpi_{p}} \varpi_{p}(\tau)-g_{\nu_{0}} \delta \hat{v}(\tau)\right] \mathbf{1}_{-} \\
+\hat{\kappa}_{0} g_{\mu_{p}} \hat{\mu}_{p}(\tau), \\
\mathbf{s}_{0}^{(2)}(\tau)=i\left[g_{\varpi_{p}} \varpi_{p} \alpha_{0}-i g_{v_{0}} \delta \hat{v} \alpha_{0}\right] \mathbf{1}_{-}-\frac{1}{2} E \hat{\kappa}_{0} \alpha^{(1) 2},
\end{gathered}
$$

with $\mathbf{1}_{-}=(1,-1)^{T}$ and

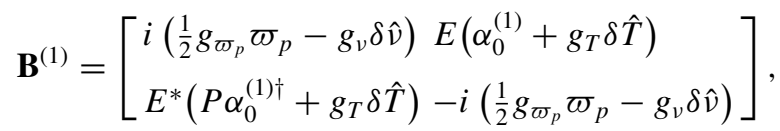

with $P$ being the permutation operator. Note that $\delta \hat{T}$ and $\alpha_{0}^{(1)}$ appear in the off-diagonal terms while $\varpi_{p}$ and $\delta \hat{v}$ are in the diagonal terms.

For a tuned OPO $\left(\psi=\psi_{0}=\phi_{\chi}=0\right), B^{(2)}, \delta B^{(2)}$, and $\mathbf{B}^{(1,1)}$, of the third equation of Eq. (10), are given by

$$
\begin{gathered}
B^{(2)}=E\left\langle\alpha_{0}^{(2)}\right\rangle=-\frac{g_{\chi}^{2}}{2\left(1-E^{2}\right)}-g_{\varpi_{p}} \frac{E}{\hat{\kappa}_{0}}-g_{v}^{2} \frac{E}{\hat{\kappa}_{0}^{2}}, \\
\delta B^{(2)}\left(\tau-\tau^{\prime}\right)=-E^{2} \hat{\kappa}_{0} G_{0}\left(\tau-\tau^{\prime}\right) \sigma_{a \alpha^{\dagger}}^{(1,1)}\left(\tau-\tau^{\prime}\right), \\
\mathbf{B}^{(1,1)}\left(\tau-\tau^{\prime}\right)=\left\langle\mathbf{B}^{(1)}(\tau) \cdot \mathbf{G}\left(\tau-\tau^{\prime}\right) \cdot \mathbf{B}^{(1)}\left(\tau^{\prime}\right)\right\rangle,
\end{gathered}
$$


with $\sigma_{a \alpha^{\dagger}}^{(1,1)}\left(\tau-\tau^{\prime}\right)=\left\langle\alpha^{(1)}(\tau) \alpha^{(1) \dagger}\left(\tau^{\prime}\right)\right\rangle$. To be consistent with the preceding hierarchy, all moments $\left\langle s^{(m)} s^{(m)}\right\rangle$ must satisfy the inequalities $\left\langle s^{(1)} s^{(1)}\right\rangle>\left\langle s^{(2)} s^{(2)}\right\rangle>\left\langle s^{(3)} s^{(3)}\right\rangle$. Close to the critical point, $\left\langle s^{(1)} s^{(1)}\right\rangle,\left\langle s^{(2)} s^{(2)}\right\rangle$, and $\left\langle s^{(3)} s^{(3)}\right\rangle$ are of the order of $O\left(g_{\chi}^{2}\right), \quad O\left[g_{\chi}^{2} g_{i}^{2}(1-E)^{-1}\right]$, and $O\left[g_{\chi}^{2} g_{i}^{4}(1-\right.$ $\left.E)^{-2}\right]$, respectively, so that the preceding condition implies that the approximation maintains its validity up to $1-E>g_{i}^{2}$.

Since the linear source for the down-converted beam [see Eq. (10a)] contains uniquely the quantum noise term, the effects of the classical fluctuations can be analyzed only going beyond the linear approximation.

\section{INTRACAVITY FIELD}

The nonlinear contribution to the intracavity field is represented by the averaged tensor product with respect to the different fluctuating parameters $g_{\iota} N_{\iota}\left(g_{\chi} N_{\chi}, g_{\chi} N_{\chi_{0}}, g_{\mu_{p}} \hat{\mu}_{p}\right.$, $g_{\varpi_{P}} \varpi_{P}, g_{v} \delta \hat{v}$, and $\left.g_{T} \delta \hat{T}\right)$ :

$$
\begin{aligned}
\sigma^{(\mathrm{NL})}(\tau) & =\left\langle\alpha(\tau) \alpha^{T}(0)\right\rangle-\left\langle\alpha^{(1)}(\tau) \alpha^{(1) T}(0)\right\rangle \\
& =\sigma^{(2,2)}(\tau)+\sigma^{(3,1)}(\tau)+\sigma^{(1,3)}(\tau) \\
& =g_{\chi}^{2} \sum_{\iota} g_{\iota}^{2} \sigma_{\iota}^{(\mathrm{NL})}(\tau) .
\end{aligned}
$$

Relevant $\sigma^{(m, n)}(\tau)$ are explicitly given by

$$
\begin{aligned}
& \boldsymbol{\sigma}^{(2,2)}(\tau)=\int_{-\infty}^{\tau} d \tau^{\prime} \int_{-\infty}^{0} d \tau^{\prime \prime} \mathbf{G}\left(\tau-\tau^{\prime}\right) \cdot\left\langle\mathbf{B}^{(1)}\left(\tau^{\prime}\right) \cdot \boldsymbol{\sigma}^{(1,1)}\left(\tau^{\prime}-\tau^{\prime \prime}\right) \cdot \mathbf{B}^{(1) T}\left(\tau^{\prime \prime}\right)\right\rangle \cdot \mathbf{G}\left(-\tau^{\prime \prime}\right) \\
& \boldsymbol{\sigma}^{(3,1)}(\tau)=\int_{-\infty}^{0} d \tau \mathbf{G}\left(\tau-\tau^{\prime}\right) \cdot\left\{B^{(2)}\left[\begin{array}{ll}
0 & 1 \\
1 & 0
\end{array}\right]+\int_{-\infty}^{\tau}\left[\delta B^{(2)}\left(\tau-\tau^{\prime}\right) \mathbf{1}+\mathbf{B}^{(1,1)}\left(\tau-\tau^{\prime}\right)\right] \cdot \boldsymbol{\sigma}^{(1,1)}\left(\tau^{\prime}\right) d \tau^{\prime}\right\}
\end{aligned}
$$

Of particular interest is the variance $\left\langle X_{\pi / 2}^{(\mathrm{NL}) 2}\right\rangle_{\iota}$ normalized to $\left\langle X_{\pi / 2}^{2}\right\rangle$, that is, the weight, normalized to $g_{\iota}^{2}$, of the nonlinear correction to the squeezed variance:

$$
\lambda_{l}^{\mathrm{NL}}=\frac{\left\langle X_{\pi / 2}^{(\mathrm{NL}) 2}\right\rangle_{\iota}}{\left\langle X_{\pi / 2}^{2}\right\rangle}=\frac{-\sigma_{\iota a a}^{(\mathrm{NL})}(0)+\sigma_{\iota a a^{\dagger}}^{(\mathrm{NL})}(0)+\sigma_{\iota a^{\dagger} a}^{(\mathrm{NL})}(0)-\sigma_{l a^{\dagger} a^{\dagger}}^{(\mathrm{NL})}(0)}{4\left\langle X_{\pi / 2}^{2}\right\rangle} .
$$

For a balanced and exactly tuned OPO, we have

$$
\begin{gathered}
\lambda_{\chi_{0}}^{\mathrm{NL}}=\frac{E\left\{2 E^{3} \hat{\kappa}_{0}+2 E^{2} \hat{\kappa}_{0}\left(2+\hat{\kappa}_{0}\right)+\left(2+\hat{\kappa}_{0}\right)^{2}-2 E\left[-2+\hat{\kappa}_{0}\left(2+\hat{\kappa}_{0}\right)\right]\right\}}{2\left(1-E^{2}\right)(1+E)\left(2+\hat{\kappa}_{0}\right)\left(2+2 E+\hat{\kappa}_{0}\right)}, \\
\lambda_{\phi_{P}}^{\mathrm{NL}} \simeq-\frac{\hat{\kappa}_{0}\left(2+\hat{\kappa}_{0}\right)-E \hat{\kappa}_{0}\left(6+\hat{\kappa}_{0}\right)+2 E^{3}\left(8+5 \hat{\kappa}_{0}\right)-2 E^{2}\left[12+\hat{\kappa}_{0}\left(9+\hat{\kappa}_{0}\right)\right]}{16\left(1-E^{2}\right) \hat{\kappa}_{0}\left(2+\hat{\kappa}_{0}\right)}, \\
\lambda_{v}^{\mathrm{NL}} \simeq-\frac{E\left[E^{3}+2 \hat{\kappa}_{0}-E^{2}\left(3+\hat{\kappa}_{0}\right)-E\left(6+\hat{\kappa}_{0}\right)\right]}{2\left(1-E^{2}\right)(1+E) \hat{\kappa}_{0}^{2}}, \\
\lambda_{T}^{\mathrm{NL}} \simeq \frac{E^{2}(1+E)^{2}-(2+E)(1-E) \hat{\kappa}_{0}^{2}}{2\left(1-E^{2}\right)(1+E) \hat{\kappa}_{0}{ }^{2}}, \\
\lambda_{\mu_{P}}^{\mathrm{NL}} \simeq \frac{2+E^{2}}{2(1+E)^{2}} .
\end{gathered}
$$

As it is apparent from the preceding formulas, the contribution of $\hat{\mu}_{p}$ remains bounded on approaching the threshold $(E \rightarrow 1)$, whereas the other terms diverge as $(1-E)^{-1}$ (see Fig. 1).

It it noteworthy that the ratio between $\lambda_{\chi_{0}}^{N L}$ and the analogous quantity $\lambda_{\chi_{0}}^{\text {PPSE }}$ calculated in Ref. [25] by means of the positive $P$ representation (PPSE) goes as $\left(\lambda_{\chi_{0}}^{\mathrm{NL}} / \lambda_{\chi_{0}}^{\mathrm{PPSE}}\right) \simeq$ $\left(\hat{\kappa}_{0}+2\right) /\left[3 \hat{\kappa}_{0}+2+O(1-E)\right]$. In the limiting case of $E \simeq 1$, the two approaches differ by a $\hat{\kappa}_{0}$-dependent factor bounded between $1 / 3$ and 1 . Such a substantial agreement between the two results in proximity of the singular point validates the use made in this article of the extended GHLE.

\section{KURTOSIS EXCESS AND COMPARISON WITH THE EXPERIMENTAL RESULTS}

The field $\alpha_{\text {out, } 1}$ outing the OPO is a function of $\alpha$, the mirror damping coefficient $\gamma_{1}$, and the corresponding input noise $\mathbf{N}_{1}$ [29]:

$$
\alpha_{\text {out }, 1}=g_{\chi} \sqrt{2 \gamma_{1}}\left(\alpha-\frac{1}{2 \hat{\gamma}_{1}} \mathbf{N}_{1}\right) .
$$

Accordingly, the generic output quadrature reads

$$
X_{\theta}=\frac{1}{g_{\chi}^{2} \sqrt{2 \gamma_{1}}} \theta^{T} \alpha_{\text {out }, 1}=X_{\theta}^{(1)}+X_{\theta}^{(2)}+\cdots,
$$

where $\theta=1 / 2\left(e^{-i \theta}, e^{i \theta}\right)$ and $X_{\theta}^{(m)}$ corresponds to $\alpha^{(m)}$. While $X_{\theta}^{(1)}$ is Gaussian, the terms $X_{\theta}^{(m>1)}$ deviate from the normal distribution.

Quadratures are detected by a balanced homodyne, and the relative current is measured by selecting a frequency $\Omega_{f}$ and an integration time $1 / \hat{\gamma}_{f}[21,29]$. Accordingly, the detector output is represented by

$$
V_{\theta}=\hat{F}_{f} X_{\theta}=\int_{-\infty}^{0} e^{\hat{\gamma}_{f} \tau^{\prime}} \cos \left(\Omega_{f} \tau^{\prime}\right) X_{\theta}\left(\tau^{\prime}\right) d \tau^{\prime}
$$



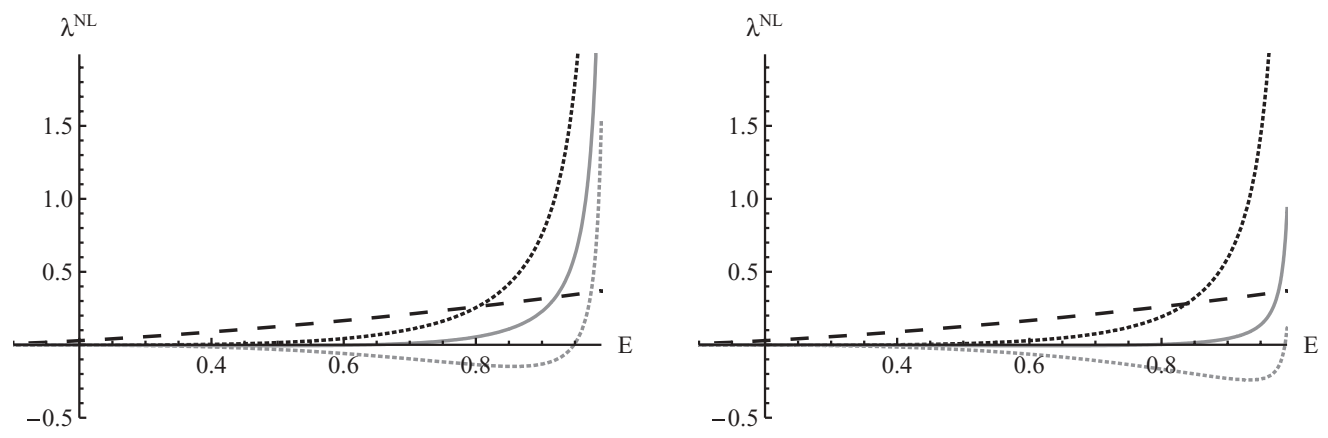

FIG. 1. Nonlinear contributions $\lambda^{\mathrm{NL}}$ [see Eq. (13)] to intracavity squeezing, as functions of the normalized pump amplitude $E$ for (left) $\hat{\kappa}_{0}=5$ and (right) $\hat{\kappa}_{0}=10$. The solid line is $\lambda_{\chi}^{\mathrm{NL}}$, the dashed line is $\lambda_{\mu}^{\mathrm{NL}}$, the dotted line is $\lambda_{\phi}^{\mathrm{NL}}$, the shaded line is $\lambda_{L}^{\mathrm{NL}}$, and the shaded dotted line is $\lambda_{T}^{\mathrm{NL}}$.

The deviation of $V_{\theta}$ from a Gaussian distribution can be measured by the kurtosis-excess parameter $K_{\theta}$ (see Appendix D for details):

$$
K_{\theta}=\frac{\left\langle: V_{\theta}^{4}:\right\rangle-3\left\langle: V_{\theta}^{2}:\right\rangle^{2}}{3\left\langle: V_{\theta}^{2}:\right\rangle^{2}} \simeq \sum_{\iota} g_{\iota}^{2} \frac{\Upsilon_{\iota}(\theta)}{\left\langle: V_{\theta}^{(1) 2}:\right\rangle_{\chi}^{2}}
$$

with $\Upsilon_{\iota}(\theta)$ given in Eq. (D1) and the weight of the different noise sources indicated generally by $N_{\iota}$.

The spectral density $\tilde{S}_{\mu_{p}}(w)=\left\langle\tilde{N}_{\mu_{p}}(-w) \tilde{N}_{\mu_{p}}(w)\right\rangle$ extends generally up to 1-2 MHz. For the sake of simplicity, it has been approximated by a uniform spectrum extending up to $1 \mathrm{MHz}$. Variables $\delta \tilde{v}$ and $\delta \tilde{T}$ extend on very narrow bandwidths, while $\tilde{N}_{\chi_{0}}$ and $\tilde{\varpi}_{p}$ are white noise sources.

In Fig. 2, we have plotted the five $\Upsilon_{\iota}(\theta)$ versus $\theta$ for three excitation strengths $E=0.71,0.87$, and 0.975 of a perfectly tuned OPO with a pump-cavity mode linewidth twice the signal one $\left(\hat{\kappa}_{0}=2\right)$, a condition similar to that of Ref. [21]. The graphs show that for $N_{\chi_{0}}, \hat{\mu}_{p}$, and $\delta \hat{T}$, the maximum deviation from a Gaussian appears for $\theta=0$, while for $\varpi_{p}$ and $\delta \hat{v}$, it occurs for $\theta= \pm \pi / 4$ [see Eq. (C2)].

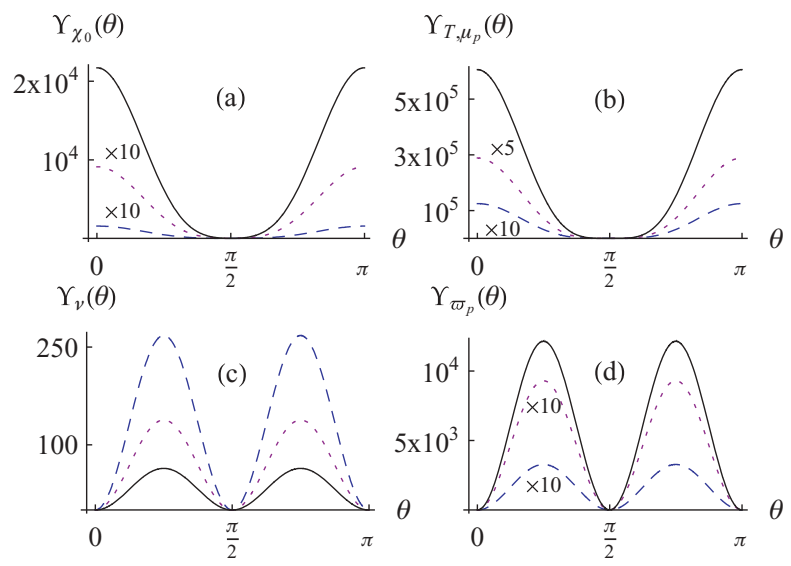

FIG. 2. (Color online) $\Upsilon_{\iota}(\theta)$ [see Eq. (D1)] vs. $\theta$ for different noise sources: (a) pump quantum noise, (b) amplitude and temperature, (c) cavity length, and (d) pump phase. The plots, referred to different scaling factors, have been calculated for typical values of $\Omega_{f}(=0.3), \gamma_{f}(=0.15)$, and $E=0.71$ (dashed blue line), 0.87 (purple dotted line), and 0.975 (solid line).
The maxima of $\Upsilon_{l}(\theta)$ for $N_{\chi_{0}}, \varpi_{p}$, and $\delta \hat{v}$ decrease for increasing $\hat{\kappa}_{0}$ (see Fig. 3). As expected from Eq. (12), the contribution of $\delta \hat{T}$ is independent of $\hat{\kappa}_{0}$. The same holds true approximately for $\hat{\mu}_{p}$ too, having considered a technical noise bandwidth that is small compared to $\hat{\kappa}_{0}$.

Looking at Fig. 3, we see that the maximum for $\Upsilon_{\mu_{p}, T}$ is at least 1 order of magnitude larger than the other ones. Moreover, $g_{\mu_{p}} \gg g_{T}$ so that for pump level up to $E^{2}=0.95$, the NG behavior is essentially because of the laser amplitude fluctuations.

In Fig. 4, $\Upsilon_{\chi_{0}}(0)$ (blue dashed line) and $\Upsilon_{\mu_{p}}(0)$ (solid line) are plotted versus $1-E^{2}$ on a double logarithmic scale. Approaching the threshold, the influence of $N_{\chi_{0}}$ increases dramatically, although $\Upsilon_{\chi_{0}}(0)$ does not overcome $\Upsilon_{\mu_{p}}(0)$. Moreover, $g_{\chi} \ll g_{\mu_{p}}$ so that the observation of pure quantum effects, predicted by Drummond et al. [25], is demanded for future technology when either new materials with huge nonlinear coefficients (enhanced $g_{\chi}$ ) or very quiet lasers (reduced $g_{\mu_{p}}$ ) will be available.

The laser amplitude noise being the prominent source influencing the nonlinear behavior, we have compared some experimental findings of Ref. [21] with the predictions of the model discussed in this article. In particular, the experimental behaviors of $K_{\theta}$ and $K_{\theta \mu_{p}}$ versus $\theta \in(-\pi, \pi)$ are reported in Fig. 5. Moreover, the maximum value of the experimental

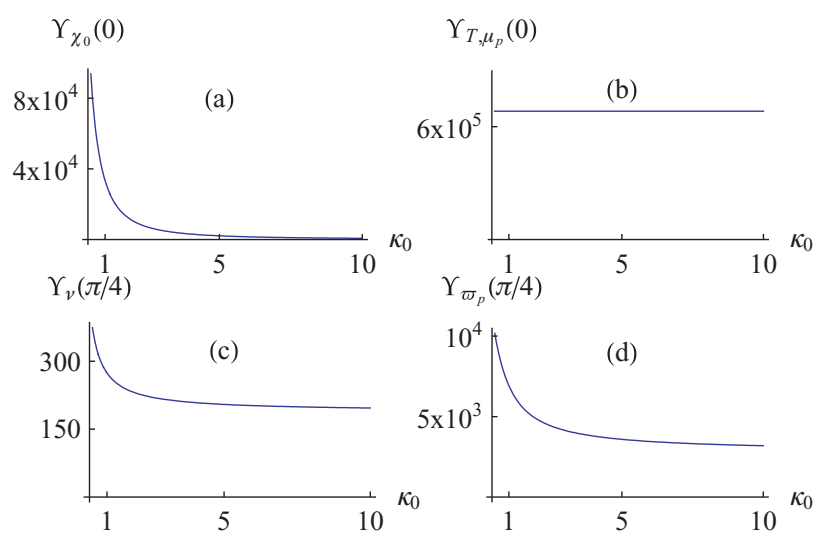

FIG. 3. (Color online) Maxima of $\Upsilon_{\iota}(\theta)$ [see Eq. (D1)] vs. $\kappa_{0}$ for the conditions of Fig. $2(E \rightarrow 0.975)$. 


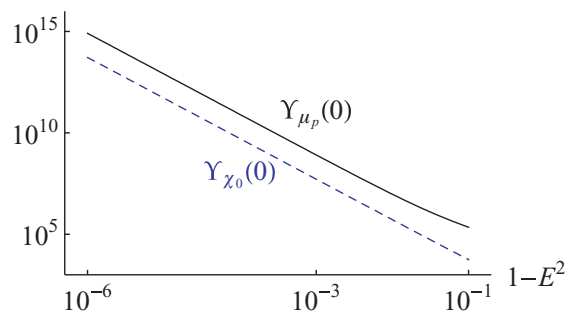

FIG. 4. (Color online) $\Upsilon_{\chi 0}(0)$ (pump quantum noise) and $\Upsilon_{\mu_{p}}(0)$ (amplitude fluctuations) vs. $1-E^{2}$.

kurtosis, for $\theta=0$, and $g_{\mu_{p}}^{2} \Upsilon_{\mu_{p}}(0)$ [see Eq. (16)] versus $E^{2} \in(0.45,0.97)$ are plotted in Fig. 6 .

In general, $\Upsilon_{\mu_{p}}(\theta)$ can be represented by

$$
\Upsilon_{\mu_{p}}(\theta)=\Upsilon_{4 \mu_{p}} \cos 4 \theta+\Upsilon_{2 \mu_{p}} \cos 2 \theta+\Upsilon_{0 \mu_{p}},
$$

with $\Upsilon_{4,2,0 \mu_{p}}$ functions of $E, \hat{\kappa}_{0}$, and $\hat{\kappa}$. For very small deviations from the resonant configuration and $|E|$ close to $1, \Upsilon_{\mu_{p}}(\theta)$ depends critically on $|E|$ :

$$
|E|=\frac{E_{0}}{\sqrt{\left(1+4 \frac{v_{p}^{2}}{\gamma_{s}^{2}}\right)\left(1+\frac{v_{p}^{2}}{\gamma_{p}^{2}}\right)}},
$$

with $E_{0}=\epsilon|\bar{\chi}| /\left(\gamma_{p} \gamma_{s}^{3 / 2}\right)$ being the excitation strength at resonance.

The different heights of the peaks in the experimental data (see Fig. 5) can be ascribed to the variation of $E$ during a measurement. The acquisition time for $V_{\theta}$ for each $\theta$ lasted about $2 \mathrm{~ms}$, implying a total $\theta$-scanning acquisition time of $200 \mathrm{~ms}$. The apparatus was equipped with a digital controller providing a crystal temperature time constant $>10^{3} \mathrm{~s}$ and a Drever-Pound system controlling the cavity tuning with a time constant $>10$ s. However, during the total acquisition time, slow drifts of the average cavity detuning $v_{p}$ can occur, thus inducing a variation of the effective excitation parameter $E$ during the scan [see Eq. (18)]. The simplest and more direct way to account for the time dependence of $v_{p}$ is to set in

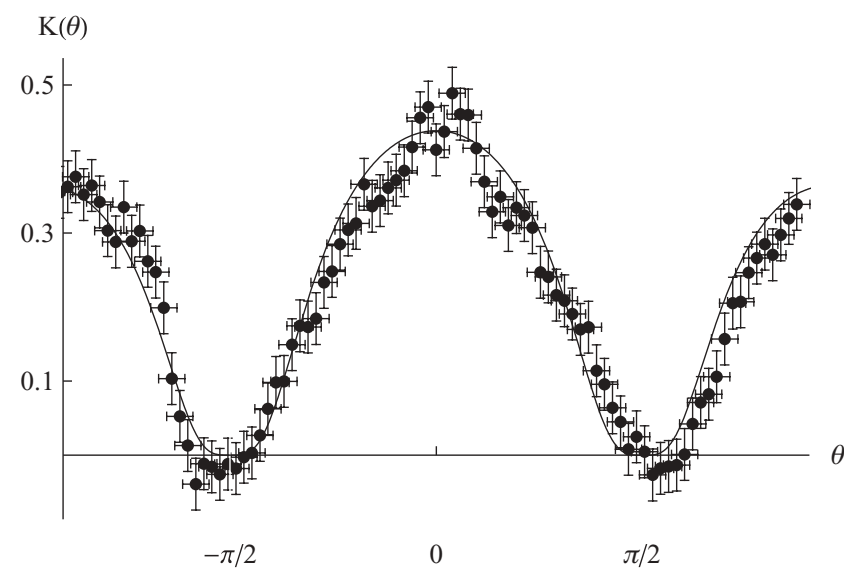

FIG. 5. $K_{\mu_{p}}(\theta)$ [see Eq. (16)] overimposed to the experimental data of Ref. [21]. $K_{\mu_{p}}(\theta)$ has been calculated by assuming $E^{2}=0.92$, $\Omega_{f}=0.3, \gamma_{f}=0.15$, and $g_{\mu_{P}}=0.007$. The horizontal error bar accounts for the detector phase $\theta$ stability, while the vertical one corresponds to the average spread between two neighbor $K_{\theta}$ data.

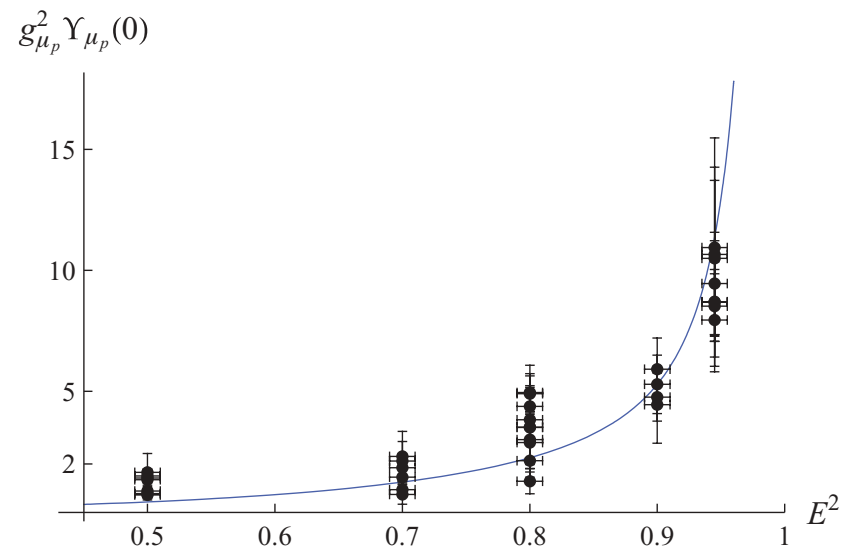

FIG. 6. (Color online) Comparison of $g_{\mu_{p}}^{2} \Upsilon_{\mu_{p}}$ (0) [Eq. (16)] vs. $E^{2}$ (solid curve; $\Omega_{f}=0.3, \gamma_{f}=0.15$, and $g_{\mu_{P}}=0.007$ ) with experimental data. Each experimental point has been obtained multiplying the measured kurtosis at $\theta=0$ for the relative squared variance. The agreement between data and the theoretical curve within error bars is evident.

Eq. (18)

$$
v_{p}=\alpha\left(\theta-\theta_{0}\right),
$$

with $\alpha$ and $\theta_{0}$ being two fitting parameters, and to express the coefficients $\Upsilon_{4,2,0 \mu_{p}}$ in Eq. (17) as functions of $\theta$.

The best fit (continuous curve of Fig. 5) of the experimental data has been computed by assuming a spectral density $\tilde{S}_{\mu_{p}}$ uniform in the interval $0-1.0 \mathrm{MHz}$, in agreement with the laser (Lightwave model 142) technical noise specification and optimizing $E_{0}$ [see Eq. (18)], $\alpha$, and $\theta_{0}$ [see Eq. (19)]. The best agreement has been obtained for $\alpha=0.013, \theta_{0}=\pi$, and $E_{0}=0.932$, values corresponding to a drift of the resonance frequency of $\approx 4 \%$ the pump cavity mode linewidth and a variation of $|E|$ of 0.006 . Finally, normalizing $g_{\mu_{p}}^{2} \Upsilon_{\mu_{p}}(\theta)$ to the squared experimental variance [see Eq. (16)], the best agreement was obtained for $g_{\mu_{p}}=0.007$, in agreement with laser noise specification $(<1 \%)$.

The NG character depends critically on the distance from the threshold (see Fig. 4); $g_{\mu_{p}}^{2} \Upsilon_{\mu_{p}}(0)$ [see Eq. (16)] versus $E^{2}$ is compared to a set of 35 data obtained for five different values of $E^{2}(0.5,0.7,0.8,0.9$, and 0.95$)$ in Fig. 6. Plotted values have been obtained multiplying the experimental kurtosis by the relative squared variance. Error bars have been obtained by standard error propagation. The good agreement between the expected behavior and the data confirms, once more, the effectiveness of the model and the validity of assumptions about the relative noise strengths.

\section{CONCLUSIONS}

The statistical properties of the fields generated by an OPO depend on the fluctuations of many classical parameters, namely, pump amplitude $\left(g_{\mu_{p}} \hat{\mu}_{p}\right)$, pump frequency $\left(g_{\varpi_{p}} \varpi_{p}\right)$, cavity detuning $\left(g_{\nu} \delta \hat{v}\right)$, and crystal temperature $\left(g_{T} \delta \hat{T}\right)$. In this article, we have presented a model of the OPO based on an extension of the GHLE that accounts for the fluctuations of these parameters. The field generated has been dealt with as 
the response of a nonlinear device to these noise sources. Then, expanding the extended GHLE system at different orders in $g_{\mu_{p}}, g_{\varpi_{p}}, g_{\nu}$, and $g_{T}$, a hierarchy of equations has been obtained, with $\hat{\mu}_{p}, \varpi_{p}, \delta \hat{v}$, and $\delta \hat{T}$ acting as noise sources, together with the quantum noises $\left(g_{\chi} \hat{N}_{\chi}, g_{\chi} \hat{N}_{\chi_{0}}\right)$ entering the optical cavity. These sources have been modeled as Gaussian processes with unit standard deviations weighted by the respective factors $g_{\mu_{p}}, g_{\varpi_{p}}, g_{v}$, and $g_{T}$, typically ranging in the intervals $10^{-2}-10^{-1}, 10^{-7 / 2}-10^{-2}, 10^{-5}-10^{-1}$, and $10^{-5}-10^{-4}$, respectively.

The extended GHLE solutions, obtained beyond the linear approximation, have been used for assessing the non-Gaussian character of the field outing a degenerate OPO. The departure of the output quadrature $X_{\theta}$ from the Gaussian statistics has been estimated by means of the kurtosis-excess figure $K_{\theta}=$ $\left(\left\langle X_{\theta}^{4}\right\rangle-3\left\langle X_{\theta}^{2}\right\rangle^{2}\right) /\left\langle X_{\theta}^{2}\right\rangle^{2}$, that is, the relative deviation of the $X_{\theta}$ fourth moment from the Gaussian expression of it. The model furnishes $K_{\theta}$ as a sum of contributions from different parameters. Sets of plots have been provided, showing the dependence of $K_{\theta}$ on the OPO operating condition, namely, the ratio pump-signal bandwidths, the excitation strength $E$, and the detection frequency $\Omega_{f}$ and bandwidth $\gamma_{f}$. For typical operating conditions, the pump technical noise emerges as the most critical factor.

The model has been used for reproducing the experimental values, reported in Ref. [21], of $K_{\theta}$ versus $\theta$ and $K_{0}$ versus $E^{2}$. The good agreement, within the error bars, of the experimental data with the analytic predictions confirms the validity of the presented model. By providing a physically insightful and computationally effective parametrization of the OPO, the model may help in addressing the generation of NG states by means of OPO sources.

\section{APPENDIX A: LINEARIZATION BELOW THRESHOLD}

The Fourier transform of the Green's functions $\mathbf{G}, \mathbf{G}_{0}$ relative to a degenerate OPO are given by

$$
\tilde{\mathbf{G}}=\frac{1}{\tilde{D}}\left[\begin{array}{cc}
\tilde{\Delta}^{\ddagger} & e^{-i \vartheta}|E| \\
e^{i \vartheta}|E| & \tilde{\Delta}
\end{array}\right], \quad \tilde{\mathbf{G}}_{0}=\left[\begin{array}{cc}
\tilde{\Delta}_{0}^{-1} & 0 \\
0 & \tilde{\Delta}_{0}^{\ddagger-1}
\end{array}\right],
$$

with $\vartheta=\psi-\frac{1}{2} \psi_{0}, \quad \tilde{\Delta}=\hat{\kappa}-i \omega, \quad \tilde{D}(\omega)=\tilde{\Delta} \tilde{\Delta}^{\ddagger}-|E|^{2}=$ $-\left(\omega+\omega_{+}\right)\left(\omega+\omega_{-}\right)$, and $\tilde{\Delta}_{0}=\hat{\kappa}_{0}-i \omega, \omega_{ \pm}=i(\cos \psi \mp$ $\left.\sqrt{|E|^{2}-\sin ^{2} \psi}\right)$. In particular, for $\hat{\kappa}=1$ (tuned device), they correspond in the time domain to

$$
\begin{aligned}
& \mathbf{G}(\tau)= \begin{cases}e^{-\tau}\left[\begin{array}{ll}
\cosh (E \tau) & \sinh (E \tau) \\
\sinh (E \tau) & \cosh (E \tau)
\end{array}\right], & \tau>0, \\
0, & \tau<0,\end{cases} \\
& \mathbf{G}_{0}(\tau)= \begin{cases}e^{-\hat{\kappa}_{0} \tau}\left[\begin{array}{ll}
1 & 0 \\
0 & 1
\end{array}\right], & \tau>0, \\
0, & \tau<0 .\end{cases}
\end{aligned}
$$

\section{APPENDIX B: TIME-NORMAL ORDERED CORRELATION MATRIX : $\tilde{\sigma}^{(1,1)}(\omega):$}

The Fourier transform : $\tilde{\sigma}^{(1,1)}(\omega)$ : of the time-normal ordered matrix : $\boldsymbol{\alpha}\left(\tau-\tau^{\prime}\right):=:\left\langle\boldsymbol{\alpha}^{(1)}(\tau) \alpha^{(1) T}\left(\tau^{\prime}\right)\right\rangle_{\chi}:\left[: \boldsymbol{\alpha}\left(\tau^{\prime}-\tau\right)\right.$ $\left.:=: \boldsymbol{\alpha}\left(\tau-\tau^{\prime}\right):\right]$ and $\left.: \boldsymbol{\alpha}_{a^{\dagger} a^{\dagger}}:=: \boldsymbol{\alpha}_{a a}^{*}:, \boldsymbol{\alpha}_{a a^{\dagger}}:=: \boldsymbol{\alpha}_{a^{\dagger} a}:\right)$ is obtained from $\tilde{\sigma}^{(1,1)}(\omega)=\tilde{\sigma}(\omega) /\left[\left(\omega^{2}-\omega_{+}^{2}\right)\left(\omega^{2}-\omega_{-}^{2}\right)\right]$ with

$$
\tilde{\sigma}(\omega)=4\left[\begin{array}{cc}
\frac{\tilde{\Delta}^{\ddagger}}{e^{-i \vartheta}|E|} & \frac{\tilde{\Delta}^{\ddagger}(-\omega) \tilde{\Delta}(\omega)}{|E|^{2}} \\
1 & \frac{\tilde{\Delta}}{e^{i \vartheta}|E|}
\end{array}\right]
$$

by first normally ordering $\tilde{\sigma}(\omega)$,

$$
\tilde{\sigma}(\omega) \rightarrow \tilde{\sigma}_{N}(\omega)=4\left[\begin{array}{cc}
\frac{\tilde{\Delta}^{\ddagger}}{e^{-i \vartheta}|E|} & 1 \\
1 & \frac{\left(\tilde{\Delta}^{\ddagger}\right)^{*}}{e^{i \vartheta}|E|}
\end{array}\right]
$$

and then symmetrizing with respect to time reversal:

$$
\begin{aligned}
: \tilde{\sigma}^{(1,1)}(-\omega): & =-\frac{2 \omega_{+}}{\omega^{2}-\omega_{+}^{2}} \tilde{\boldsymbol{\sigma}}_{N}\left(-\omega_{+}\right)-\frac{2 \omega_{-}}{\omega^{2}-\omega_{-}^{2}} \tilde{\boldsymbol{\sigma}}_{N}\left(-\omega_{-}\right) \\
& =\frac{\tilde{\sigma}_{T N}(\omega)}{\left(\omega^{2}-\omega_{+}^{2}\right)\left(\omega^{2}-\omega_{-}^{2}\right)} .
\end{aligned}
$$

In particular, for the tuned case,

$$
\tilde{\sigma}_{T N}(\omega)=4\left[\begin{array}{cc}
\frac{1+\mathrm{E}^{2}+\omega^{2}}{2 E} & 1 \\
1 & \frac{1+\mathrm{E}^{2}+\omega^{2}}{2 E}
\end{array}\right]
$$

\section{APPENDIX C: $B^{(1)}$ EXPANSION}

The different noise sources $N_{\iota}$ contribute to $\tilde{\mathbf{B}}^{(1)}$ [Eq. (12)] through the terms

$$
\tilde{\mathbf{B}}^{(1)}=g_{\chi} \tilde{N}_{\chi_{0}} \tilde{\mathbf{B}}_{\chi}^{(1)}+g_{\chi} \tilde{N}_{\chi 0}^{\ddagger} \tilde{\mathbf{B}}_{\chi}^{(1) T}+\sum_{i} g_{i} \tilde{N}_{i} \tilde{\mathbf{B}}_{i}^{(1)},
$$

with

$$
\begin{gathered}
\tilde{\mathbf{B}}_{\chi}^{(1)}=E\left[\begin{array}{ll}
0 & \tilde{\Delta}_{0}^{-1} \\
0 & 0
\end{array}\right], \\
\tilde{\mathbf{B}}_{\mu_{P}}^{(1)}=\left[\begin{array}{ll}
0 & e^{-i \vartheta}|E| \hat{\kappa}_{0} \tilde{\Delta}_{0}^{-1} \\
e^{i \vartheta}|E| \hat{\kappa}_{0}^{*} \tilde{\Delta}_{0}^{\ddagger-1} & 0
\end{array}\right], \\
\tilde{\mathbf{B}}_{\omega_{P}}^{(1)}=i\left[\begin{array}{ll}
\frac{1}{2} & e^{-i \vartheta}|E| \tilde{\Delta}_{0}^{-1} \\
-e^{i \vartheta}|E| \tilde{\Delta}_{0}^{\ddagger-1} & -\frac{1}{2}
\end{array}\right], \\
\tilde{\mathbf{B}}_{T}^{(1)}=\left[\begin{array}{ll}
0 & e^{-i \vartheta}|E| \\
e^{i \vartheta}|E| & 0
\end{array}\right], \\
\tilde{\mathbf{B}}_{v}^{(1)}=i\left[\begin{array}{ll}
-1 & e^{-i \vartheta}|E| \tilde{\Delta}_{0}^{-1} \\
-e^{i \vartheta}|E| \tilde{\Delta}_{0}^{\ddagger-1} & 1
\end{array}\right] .
\end{gathered}
$$

\section{APPENDIX D: KURTOSIS-EXCESS EXPANSION}

From the vanishing of the time-normal ordered correlations $\left\langle: \alpha^{(1)} N_{1}^{T}:\right\rangle=\left\langle: \alpha^{(2)} N_{1}^{T}:\right\rangle=0$, it follows that

$$
\left\langle: X_{\theta}^{(l)} X_{\theta}^{(m)}:\right\rangle_{\chi}=\theta^{T} \cdot\left\langle: \alpha^{(l)} \alpha^{(m) T}:\right\rangle_{\chi} \cdot \theta
$$

with $l, m=1,2$. Hence, retaining only the lowest nonlinear orders for $\left\langle: V_{\theta}^{4}:\right\rangle$ and $\left\langle: V_{\theta}^{2}:\right\rangle^{2}, \Upsilon_{\iota}(\theta)$ [see Eq. (16)] reads

$$
\begin{aligned}
\Upsilon_{\iota}(\theta) & =\left\langle\left(\left\langle: V_{\theta}^{(1)} V_{\theta \iota}^{(2)}+V_{\theta \iota}^{(2)} V_{\theta}^{(1)}:\right\rangle_{\chi}\right)^{2}\right\rangle_{\iota} \\
& =\frac{1}{2 \pi} \int_{-\infty}^{\infty} \tilde{S}_{\iota}(w) \theta^{T} \cdot \tilde{\varsigma}_{\iota}(-w) \cdot \boldsymbol{\theta} \boldsymbol{\theta}^{T} \cdot \tilde{\varsigma}_{\iota}(w) \cdot \theta d w \\
& =\Upsilon_{4 \iota} \cos 4 \theta+\Upsilon_{2 \iota} \cos 2 \theta+\Upsilon_{0 \iota},
\end{aligned}
$$


with $\tilde{S}_{\iota}(w)=\left\langle\tilde{N}_{i}(-w) \tilde{N}_{i}(w)\right\rangle$ and $\tilde{\zeta}_{\iota}$ being a $2 \times 2$ matrix

$$
\begin{aligned}
\tilde{\zeta}_{\iota}(w) & =(1+\hat{T})\left\langle:\left(\hat{F}_{f} \alpha_{\iota}^{(2)}(0)\right) \hat{F}_{f} \alpha^{(1) T}(0):\right\rangle_{\chi} \\
& =\frac{1}{2 \pi} \frac{1}{\hat{\kappa}_{0}-i w} \int_{-\infty}^{\infty} \tilde{H}(w, \omega) \tilde{\boldsymbol{\sigma}}_{\iota}(w, \omega) d \omega ;
\end{aligned}
$$

$\hat{T}$ being a matrix transposition operator; $\tilde{\boldsymbol{\sigma}}_{\iota}(w, \omega)$ being an entire function of $\omega$ and $w$,

$$
\begin{aligned}
\tilde{\boldsymbol{\sigma}}_{\iota}(w, \omega)= & (1+\hat{T})\left(\hat{\kappa}_{0}-i w\right) \tilde{D}(w+\omega) \\
& \times \tilde{\boldsymbol{G}}(w+\omega) \cdot \tilde{\mathbf{B}}_{\iota}^{(1)}(w) \cdot \tilde{\boldsymbol{\sigma}}_{T N}(\omega),
\end{aligned}
$$

with $\tilde{\boldsymbol{\sigma}}_{T N}(\omega)$ defined in Eq. (B1) as

$$
\tilde{H}(w, \omega)=\frac{\tilde{F}_{f}(\omega+w) \tilde{F}_{f}(-\omega)}{\tilde{D}(w+\omega)\left(\omega^{2}-\omega_{+}^{2}\right)\left(\omega^{2}-\omega_{-}^{2}\right)} ;
$$

and

$$
\tilde{F}_{f}(\omega)=\frac{i}{2}\left(\frac{1}{\omega-\Omega_{-}}+\frac{1}{\omega-\Omega_{+}}\right)
$$

being the Fourier transform of $\hat{F}_{f}$ [see Eq. (15)], while $\Omega_{ \pm}=$ $\pm \Omega_{f}-i \gamma_{f}$.

Since $\lim _{|\omega| \rightarrow \infty} \omega \tilde{H}(w, \omega) \tilde{\boldsymbol{\sigma}}_{\iota}(w, \omega)=0$, the right-hand side of Eq. (D2) is given by the sum of residues

$$
\tilde{\zeta}_{\iota}(w)=\frac{i}{\hat{\kappa}_{0}-i w} \sum_{l=1}^{4} H^{(l)}(w) \tilde{\boldsymbol{\sigma}}_{\iota}\left(w, \omega_{l}\right),
$$

where $H^{(l)}(w)=\operatorname{Res}_{\omega=\omega_{l}}[H(w, \omega)]$ for $\omega_{l}=\omega_{+}, \omega_{-},-\Omega_{+}$, and $-\Omega_{-}(l=1,2,3,4)$ poles of $\tilde{H}(w, \omega)$ in the upper complex $\omega$ plane.
In the limiting case of zero-centered $\delta$-like sources $\tilde{N}_{i}$ [see Eq. (C1)],

$$
\Upsilon_{i}(\theta)=\theta^{T} \cdot \tilde{\zeta}_{i}(0) \cdot \boldsymbol{\theta} \boldsymbol{\theta}^{T} \cdot \tilde{\zeta}_{i}(0) \cdot \theta .
$$

Such an approximation holds true for $\tilde{N}_{T}, \tilde{N}_{v}$, and in a lesser measure for $\tilde{N}_{\mu_{p}}$, depending on the laser technical noise bandwidth normalized to the OPO cavity one. At the other extreme, $\tilde{N}_{\chi_{0}}$ and $\tilde{N}_{\varpi_{p}}$ represent white noises processes for which $\Upsilon_{\chi_{0}, \varpi_{p}}(\theta)$ reduce to

$$
\begin{gathered}
\Upsilon_{\chi_{0}, \varpi_{p}}(\theta)=-\sum_{l=1}^{4} H^{(l)}\left(-i \hat{\kappa}_{0}\right) \\
\boldsymbol{\theta}^{T} \cdot \boldsymbol{\sigma}_{\chi_{0}, \varpi_{p}}\left(-i \hat{\kappa}_{0}, \omega_{l}\right) \cdot \boldsymbol{\theta} \boldsymbol{\theta}^{T} \cdot \tilde{\boldsymbol{\zeta}}_{\chi_{0}, \varpi_{p}}\left(i \hat{\kappa}_{0}\right) \cdot \boldsymbol{\theta} \\
-\sum_{l, i=1}^{4} \frac{1}{\hat{\kappa}_{0}+i w_{i}^{(l)}} \operatorname{Res}_{w=-w_{i}^{(l)}}\left[H^{(l)}(w)\right] \\
\boldsymbol{\theta}^{T} \cdot \tilde{\boldsymbol{\sigma}}_{\chi_{0}, \varpi_{p}}\left(-w_{i}^{(l)}, \omega_{l}\right) \cdot \boldsymbol{\theta} \boldsymbol{\theta}^{T} \cdot \tilde{\zeta}_{\chi_{0}, \varpi_{p}}\left(w_{i}^{(l)}\right) \cdot \boldsymbol{\theta},
\end{gathered}
$$

with $\omega_{l}$ being the frequencies of (D3) and $w_{i}^{(l)}$ the poles of $H^{(l)}(w)$ in the upper complex $w$ plane:

$$
\begin{gathered}
w_{i}^{(1)}=\left\{2 \omega_{+}, \omega_{+}-\Omega_{+}, \omega_{+}-\Omega_{-}, \omega_{+}+\omega_{-}\right\}, \\
w_{i}^{(2)}=\left\{2 \omega_{-}, \omega_{-}-\Omega_{-}, \omega_{-}-\Omega_{+}, \omega_{-}+\omega_{+}\right\}, \\
w_{i}^{(3)}=\left\{-2 \Omega_{+}, \omega_{+}-\Omega_{+}, \omega_{-}-\Omega_{+},-\Omega_{+}-\Omega_{-}\right\}, \\
w_{i}^{(4)}=\left\{-2 \Omega_{-}, \omega_{-}-\Omega_{-}, \omega_{+}-\Omega_{-},-\Omega_{-}-\Omega_{+}\right\} .
\end{gathered}
$$

[1] J. Eisert, S. Scheel, and M. B. Plenio, Phys. Rev. Lett. 89, 137903 (2002).

[2] J. Fiurášek, Phys. Rev. Lett. 89, 137904 (2002).

[3] D. E. Browne, J. Eisert, S. Scheel, and M. B. Plenio, Phys. Rev. A 67, 062320 (2003).

[4] A. Franzen, B. Hage, J. Di Guglielmo, J. Fiurášek, and R. Schnabel, Phys. Rev. Lett. 97, 150505 (2006).

[5] J. Fiurášek, P. Marek, R. Filip, and R. Schnabel, Phys. Rev. A 75, 050302(R) (2007).

[6] T. Kiesel, W. Vogel, B. Hage, J. Di Guglielmo, A. Samblowski, and R. Schnabel, Phys. Rev. A 79, 022122 (2009).

[7] C. H. Bennett, D. P. DiVincenzo, J. A. Smolin, and W. K. Wootters, Phys. Rev. A 54, 3824 (1996).

[8] J.-W. Pan, C. Simon, C. Brukner, and A. Zeilinger, Nature 410, 1067 (2001).

[9] H. Aschauer and H. J. Briegel, Phys. Rev. Lett. 88, 047902 (2002); Phys. Rev. A 66, 032302 (2002).

[10] H. Takahashi, J. S. Neergaard-Nielsen, M. Takeuchi, M. Takeoka, K. Hayasaka, A. Furusawa, and M. Sasaki, Nature Photonics 4, 178 (2010).

[11] T. Opatrný, G. Kurizki, and D.-G. Welsch, Phys. Rev. A 61, 032302 (2000).

[12] P. T. Cochrane, T. C. Ralph, and G. J. Milburn, Phys. Rev. A 65, 062306 (2002).
[13] S. Olivares, M. G. A. Paris, and R. Bonifacio, Phys. Rev. A 67, 032314 (2003); S. Olivares and M. G. A. Paris, Las. Phys. 16, 1533 (2006).

[14] N. J. Cerf, O. Krüger, P. Navez, R. F. Werner, and M. M. Wolf, Phys. Rev. Lett. 95, 070501 (2005).

[15] S. Olivares and M. G. A. Paris, Phys. Rev. A 70, 032112 (2004).

[16] S. Olivares and M. G. A. Paris, J. Opt. B: Quantum Semiclass. Opt. 7, S392 (2005).

[17] A. Ferraro and M. G. A. Paris, J. Opt. B: Quantum Semiclass. Opt. 7, 174 (2005).

[18] C. Invernizzi, S. Olivares, M. G. A. Paris, and K. Banaszek, Phys. Rev. A 72, 042105 (2005).

[19] J. Wenger, R. Tualle-Brouri, and P. Grangier, Phys. Rev. Lett. 92, 153601 (2004); A. Alexei Ourjoumtsev, R. Tualle-Brouri, J. Laurat, and P. Grangier, Science 312, 83 (2006); J. Heersink, C. Marquardt, R. Dong, R. Filip, S. Lorenz, G. Leuchs, and U. L. Andersen, Phys. Rev. Lett. 96, 253601 (2006).

[20] A. Zavatta, S. Viciani, and M. Bellini, Science 306, 660 (2004).

[21] V. D'Auria, A. Chiummo, M. De Laurentis, A. Porzio, S. Solimeno, and M. G. A. Paris, Opt. Express 13, 948 (2005); J. Řeháĉek, S. Olivares, D. Mogilevtsev, Z. Hradil, M. G. A. Paris, S. Fornaro, V. D'Auria, A. Porzio, and S. Solimeno, Phys. Rev. A 79, 032111 (2009). 
[22] P. D. Drummond and P. Kinsler, Quantum Semiclass. Opt. 7, 727 (1995); P. Kinsler and P. D. Drummond, Phys. Rev. A 52, 783 (1995).

[23] S. F. Pereira, Z. Y. Ou, and H. J. Kimble, Phys. Rev. A 62, 042311 (2000).

[24] M. D. Reid and P. D. Drummond, Phys. Rev. Lett. 60, 2731 (1988); Phys. Rev. A 40, 4493 (1989); P. D. Drummond and M. D. Reid, ibid. 41, 3930 (1990).

[25] S. Chaturvedi, K. Dechoum, and P. D. Drummond, Phys. Rev. A 65, 033805 (2002); P. D. Drummond, K. Dechoum, and S. Chaturvedi, ibid. 65, 033806 (2002); K. Dechoum, P. D. Drummond, S. Chaturvedi, and M. D. Reid, ibid. 70, 053807
(2004); S. Chaturwedi and P. D. Drummond, Eur. Phys. J. B 8, 251 (1999).

[26] R. Graham and H. Hake, Z. Phys. 210, 276 (1968); R. Graham, ibid. 210, 319 (1968).

[27] G. D. Boyd and D. A. Kleinman, J. Appl. Phys. 39, 3597 (1968); Y. F. Chen and Y. C. Chen, Appl. Phys. B 76, 645 (2003).

[28] M. J. Collett and C. W. Gardiner, Phys. Rev. A 30, 1386 (1984); C. W. Gardiner and M. J. Collett, ibid. 31, 3761 (1985); M. J. Collett and D. F. Walls, ibid. 32, 2887 (1985).

[29] L. A. Wu, H. J. Kimble, J. L. Hall, and H. Wu, Phys. Rev. Lett. 57, 2520 (1986). 